\title{
Qualification of High Heat Flux Components: Application to Target Elements of W7-X Divertor
}

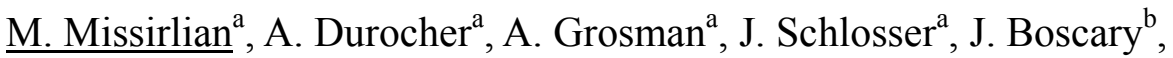 \\ F. Escourbiac ${ }^{\mathrm{a}}$, F. Cismondi ${ }^{\mathrm{a}}$ \\ ${ }^{a}$ Association Euratom-CEA, CEA/DSM/DRFC, CEA Cadarache, F-13108 Saint Paul Lez Durance, France \\ ${ }^{b}$ Max-Planck-Institut für Plasmaphysik, EURATOM Association, 85748 Garching, Germany
}

\begin{abstract}
The development of actively cooled plasma-facing components (PFC) represents one of fusion's most challenging engineering efforts. In this frame, a high-quality bonding between the refractory armour and the heat sink is essential to ensure the heat removal capability and the thermal performances of PFC. Experience gained during manufacturing of Tore Supra actively cooled PFC led to the establishment of a qualification methodology and provided a large experience of acceptance criteria using an active infrared thermography (SATIR). This paper presents the application of this qualification process to the $\mathrm{W} 7-\mathrm{X}$ pre-series components, with the objective of assessing and defining workable acceptance criteria that enable reliable predictions of performance at the nominal heat flux requirements in W7-X. Finally, to check the reliability of the non-destructive examination (NDE) method by transient infrared thermography, the newly defined acceptance criteria were applied to W7-X pre-series target elements (Batch \#3). The SATIR results, benchmarked with HHF tests performed on the GLADIS ion beam facility were discussed to assess the ability to detect critical defects at the interface between tiles and heat sink.
\end{abstract}

\section{Introduction}

Modern steady state magnetic fusion devices require the use of actively cooled high heat flux (HHF) plasma-facing components (PFC) to handle heat fluxes from the plasma in the range of $10-20 \mathrm{MW} / \mathrm{m}^{2}$. This generates a number of engineering constraints: the cooling system -which is generally based on a circulation of pressurized water into the HHF PFC's heat sink- must offer high thermal efficiency, the heat sink must offer high thermal conductivity and high mechanical resistance, the armour materials must be refractory and compatible with plasma wall interaction (low sputtering and/or low atomic number). Furthermore, the assembling of the refractory armour material onto the structural metallic heat sink causes generic difficulties mainly associated with their thermal expansion mismatch.

As heat exhaust capability and lifetime of PFC during in-situ operation are linked to the manufacturing quality, a set of qualification activities is to be performed throughout all the component development phases following a qualification methodology. High heat flux (HHF) testing is the central activity of this method, it allows to check the robustness of the PFC in real thermal conditions, to evaluate the limit of the design, to give information on fatigue lifetime and to check the reliability of the Non-Destructive Examination (NDE) techniques. The ultimate objective is to have a qualified non-destructive method to be applied in serial production that is a good prediction of performance under nominal heat flux.

Based on success of the qualification route, which has been followed to define an acceptance criterion for flat tile PFC joining for Tore Supra Toroidal Pumped Limiter (TPL) series production, this paper presents the progress in application of this methodology applied to the 
definition of workable acceptance criteria for the CFC flat tile target elements of the W7-X divertor.

\section{Qualification Methodology}

A large experience has been gained at Tore Supra for actively cooled PFC working at steady state since 1989 and especially since 2002 in the frame of the CIEL project (French acronym for Internal Components and Limiters) [1]. This pioneering activity developed by CEA and the European industry in the field of actively cooled HHF PFC, which is today culminating with the routine operation of an actively cooled toroidal pumped limiter (TPL) capable of sustaining heat fluxes up to $10 \mathrm{MW} / \mathrm{m}^{2}$, represents a world-wide unique example of series production of HHF components. Many lessons were learnt from this experience throughout all the component development phases. The qualification methodology is based on a design by experiment approach, whereby a design or designs developed through modelling are manufactured and tested using non-destructive examination techniques and HHF testing, and finally post-mortem destructive analysis. Based on this experience, the designs are then improved, again aided by modelling. Several iterations may be needed to arrive at an acceptable design. This approach has been adopted by Tore Supra, W7-X and ITER for their PFC development programmes [2, 3]. Even though a large number of difficulties appear unavoidable during the qualification activity, one major and recurrent technical issue that has to be solved is the assembly of a "plasma compatible" armour to a heat sink which can provide both the heat exhaust through pressurized water and mechanical integrity. HHF testing is the central activity of this method needed to guarantee a reliable detection of defects generated during PFC manufacturing, but is also time and cost consuming. Consequently, the development of non-destructive inspection methods was identified as a necessity. Based on an active infrared thermography, a dedicated NDE method was developed for this purpose and represents, associated with HHF testing, one the key means during this qualification activity, to achieve a high level of quality in the production of HHF components.

From the successful achievement of actively cooled PFC on Tore Supra, this qualification methodology was applied for W7-X Divertor and ITER Divertor vertical targets. The recent application to the W7-X pre-series components, and the progress in defining workable acceptance criteria to be applied during the serial production is presented hereafter.

\section{Application to the W7-X pre-series target elements}

The target elements of the Wendelstein 7-X (W7-X) divertor are designed to sustain a stationary heat flux of $10 \mathrm{MW} / \mathrm{m}^{2}$ and to remove a maximum power of $100 \mathrm{~kW}$. The fabrication of these HHF components (namely, 890 target elements representing several tens of thousands of $\mathrm{CFC}$ to $\mathrm{CuCrZr}$ joints) constitutes a highly demanding task. The design, the manufacturing route and the qualified non-destructive examinations are based essentially on the experience gained during EFDA R\&D activities and during the fabrication of the TPL of Tore Supra. From there, the main purposes of the pre-series phase (started in 2005) are to qualify the different steps of the fabrication, to assess the safety margin in the design and to check the reliability of NDE methods. NDE based on thermography methods are applied before ("prediction mode") and after ("correlation mode") HHF testing. It is intended by this way to evaluate and define workable acceptance criteria to be applied during the series production. 
The methodology, proposed for the qualification of W7-X divertor components, is mainly based on a cross-checking analysis between the high heat flux performance tests and the nondestructive infrared thermography examination.

\subsection{Target element design}

The highly heat-loaded area of the target plates of the W7-X divertor [4] spans a surface of $19 \mathrm{~m}^{2}$. It is formed by 890 water-cooled target elements of 14 different types. Each target element (Fig. 1) is composed of 3D carbon fibre reinforced carbon composite tiles (CFC grade Sepcarb $^{\circledR}$ NB31) assembled by $\mathrm{AMC}^{\circledR}$ (Active Metal Casting) process to OFHC copper interlayer, and joined afterwards to a heat sink made of $\mathrm{CuCrZr}$ copper alloy. The cooling geometry is formed by four straight channels equipped with twisted tapes as turbulence promoters and connected by U-bends [5]. The type of target element is characterized by its length (ranging from 250 to $595 \mathrm{~mm}$ ), and by its width (ranging from 50 to $61.5 \mathrm{~mm}$ ). For some element types, an additional width of $4.5 \mathrm{~mm}$ (so-called large side) has been introduced in the design (dashed line on the right side of the transversal view, see bottom part of Fig. 1) to allow the installation of diagnostics.

33 full-scale pre-series target elements have been delivered by Plansee SE. The first set of 11 elements (Batch \#1 and \#2) aimed to illustrate the main difficulties of the fabrication of the various geometries of target elements. In addition, dedicated probes and elements, some including artificial defects of different sizes and geometries, have been manufactured to calibrate and to qualify the NDE method during the fabrication. The second set consisted of 22 elements (Batch \#3) of the same and the simplest geometry: $200 \mathrm{~mm}$ long, $55-57 \mathrm{~mm}$ wide, 10 CFC NB31 tiles. This batch aimed to validate the CFC NB31 material for the serial fabrication and allowed to assess the accuracy and the reliability of acceptance criteria newly defined.

\subsection{Qualification of a NDE method}

The overall goal of the non-destructive testing is to demonstrate that critical defects (i.e. defects which could impair the thermal fatigue lifetime) can be reliably identified without recourse to performing high heat flux screening tests on the entire surface of each target element. This activity, performed in the frame of the pre-series phase, was carried out according to the following steps: (i) manufacture of target element prototypes including some elements with artificial calibrated defects; (ii) blind non-destructive examination of each prototype in SATIR test bed to qualify the inspection procedure; (ii) HHF tests in Judith electron beam (Batch \#1\& \#2) and GLADIS ion beam (Batch \#3) facilities to assess the behaviour of prototypes and to define acceptance criteria to be applied in serial phase; (iii) post-fatigue non-destructive examination; (iv) post-testing destructive examination.

\subsubsection{Transient thermography testing (SATIR)}

With Tore Supra TPL, CEA acquired a large experience in the inspection and acceptance processes of actively-cooled HHF elements armoured with CFC tiles using the infrared thermography SATIR (infrared test bed at CEA). The testing protocol is based on a thermal transient induced by an alternative hot/cold water flow in the heat sink structure. The design of the system allows to control several elements in parallel where the surface temperature of each tile of the elements is monitored by an infrared camera. The tile surface temperature transients are compared with those of an assumed defect free element (so-called reference) and the maximum of the temperature difference (so-called DTref_max) is stored for each tile [6]. A bonding flaw between heat sink and armour will be detected by a high value of DTref_max. It gives quantitative information on the global thermal efficiency of the component prior to its installation into the vacuum vessel of a fusion machine. This facility 
permits, also through an acceptance test based on a reference element, the detection of variability within the produced elements.

\subsubsection{Results of NDE performed on mock-ups with artificial defects}

Two mock-ups (no. 4B-1V and no. 4B-7V) with artificial calibrated defects were manufactured during the pre-series phase and controlled on SATIR before high heat flux testing (pre-HHF) [7]. The geometry and location of these artificial defects was based on the experience gained during the fabrication of the target elements: defects always located at the lateral edge of the elements, corner or strip geometries. The defects were manufactured in $\mathrm{CFC}$ at the interface between CFC and the copper interlayer, except for tile 1 at the interface between copper and $\mathrm{CuCrZr}$.

Fig. 2 shows an IR image of the DTref_max value cartography during the cooling phase for the $4 \mathrm{~B}-1 \mathrm{~V}$ target element. The main outcomes point out that strip defects up to $4 \mathrm{~mm}$ and corner defects up to $6 \times 6 \mathrm{~mm}$ are reliably detectable with SATIR. The correlation between the experimental measurements and the defect size is defined by the graphs which gives the measured DTref_max as a function of the size of the calibrated defects. Thereafter, the measurements performed on theoretically defect-free tiles allowed the evaluation of the limit of SATIR sensibility, namely $1.5^{\circ} \mathrm{C}$ for the small side (to be applied to each side of the standard elements) and $2^{\circ} \mathrm{C}$ for the large side (to be applied to one side of the diagnostic elements). The thermal-hydraulic conditions used during the SATIR tests are: water velocity $8 \mathrm{~m} / \mathrm{s}$, pressure 10 bars.

\subsubsection{Results of HHF tests performed on the pre-series elements}

HHF testing performed on pre-series target element representing the central activity in the qualification of the manufacturing process, allowed to check the robustness of the target elements to achieve a sufficiently high manufacturing quality together with a high lifetime during operation. In addition, this experimental campaign allowed the evaluation of the limit of the design and to check the reliability of the NDE method by infrared thermography SATIR.

The main conclusions are:

- No difference has been noticed in the thermal performances between the EBW and HIP technologies (i.e. AMC ${ }^{\circledR}$-NB31 tiles - CuCrZr heat sink bonding);

- At the interface between CFC and the copper interlayer, artificial defects with a size of $4 \mathrm{~mm}$ (strip defect) and of $6 \mathrm{~mm}$ (corner defect) have been systematically detected at low power density $\left(\sim 6 \mathrm{MW} / \mathrm{m}^{2}\right)$;

- No detachment of tiles but crack initiation/propagation has been observed on some tiles after a few cycles under normal operation (i.e. $\sim 10 \mathrm{MW} / \mathrm{m}^{2}$ ). This behaviour did not allow to validate fully the bonding quality CFC NB31 tiles - CuCrZr heat sink and led to the recommendations of an improvement to increase the safety margin in design before the serial production;

- SATIR pre-examination (pre-HHF) did not predict such an amount of defective tiles during the cycling HHF tests, but the post-examination (post-HHF) seems to confirm this trend under HHF. Post-testing analyses are in progress and should bring a better understanding of the damage.

\subsubsection{Correlation of SATIR NDE - HHF tests/Definition of workable acceptance criteria}

The results of SATIR pre-examination performed on target elements with artificial calibrated defects, were compared with HHF tests to correlate the joint defect size (i.e. the $\mathrm{CFC} / \mathrm{Cu}$ topology defects) detected by active infrared thermography to the thermal response obtained under heat loading. HHF testing was carried out in JUDITH electron beam facility [8] with 
different experimental conditions to achieve the nominal heat flux expected during operation in W7-X. The ultimate aim is to identify the maximum acceptable defect size (i.e. the largest defect that is stable under normal operation) and to define workable acceptance criteria for the armour joints subjected to the SATIR test.

It is assumed that the definition of an acceptance limit on the SATIR test bed is equivalent to accept e.g. a limited overheating under normal operation $\left(10 \mathrm{MW} / \mathrm{m}^{2}\right.$ in steady state). A maximum local overheating (i.e. a local increase of surface temperature with respect to the average temperature) limited to $30 \%$ for the small side and $40 \%$ for the large side with respect to the average temperature was evaluated from experimental observations in JUDITH. With these limits, the overheating of the tiles surface should have a steady state temperature smaller than $1500^{\circ} \mathrm{C}$ during operation. Finally, the maximal acceptable defect, correlated to its response under infrared examination, allows the definition of a cut-off value in terms of DTref_max on SATIR test bed (Fig. 3). Taking into account the uncertainties due to the background noise of the SATIR device and industrial factors such as materials properties and/or geometrical tolerances, this cut-off value determined for each type of target element (namely, the standard and the diagnostic elements) allows the definition of workable acceptance criteria to be applied on flat tiles with two threshold values (Fig. 4):

- The first one is the threshold value below which the examined tile is accepted without any question (color-code: white);

- The second one is the threshold value above which the examined tile is rejected, or proposed to be repaired (color-code: black);

- Between the two threshold values (color-code: grey), the examined tile is questionable and a complementary inspection method can be performed to help the decision.

\subsection{Application to pre-series target elements (Batch \#3)}

To check the reliability of the NDE method by transient infrared thermography, the newly defined acceptance criteria were applied to W7-X pre-series target elements (Batch \#3). The SATIR pre-examination results were benchmarked with HHF tests to assess the ability to detect defects at the interface between tiles and heat sink. Furthermore, SATIR postexamination (i.e after HHF testing) were also performed to analyse damage detection and propagation.

\subsubsection{SATIR pre-examination}

15 pre-series target elements of type $4 \mathrm{~S}$ with $10 \mathrm{CFC}$ tiles per elements, i.e. a maximum total amount of 150 tiles, were inspected on SATIR test bed, screened at $\sim 6 \mathrm{MW} / \mathrm{m}^{2}$ on GLADIS facility [9], then fatigued at $\sim 10 \mathrm{MW} / \mathrm{m}^{2}$ (10 seconds pulse-duration per cycle) up to 100 cycles.

Firstly, as regards the statistical distribution of DTref_max per tile (approximation by a normal distribution), a mean value $(\mu)$ close to $\sim 0.5^{\circ} \mathrm{C}$ was observed, with a standard deviation $(\sigma)<1.5^{\circ} \mathrm{C}$ (Fig. 5). This result indicates that the average quality of Batch $\# 3$ (i.e. population of 150 tiles) is reasonable in regard to the cut-off value of $4^{\circ} \mathrm{C}$ defined as the acceptance criterion for standard elements in SATIR. Correlation between the SATIR preexamination and the initial screening GLADIS tests is acceptable and reaches $90 \%$ (Fig. 7, Cycle 1). On the other hand, the correlation between failure (i.e. progressing hot spot) under thermal-cycling and initial screening is low. Large defects at the bond, which appeared during the first HHF cycles, were predicted by the SATIR pre-examination (i.e. DTref_max $>$ $4.5^{\circ} \mathrm{C}$ ). For tiles fulfilling the acceptance criteria associated to SATIR (i.e. DTref_max < $3.5^{\circ} \mathrm{C}$ ), about $28 \%$ (i.e. $\sim 40$ tiles) showed some degradation during the fatigue tests. Among these, only one tile was identified as suspicious during GLADIS screening at $\sim 6 \mathrm{MW} / \mathrm{m}^{2}$. 


\subsubsection{SATIR post-examination}

As regards the statistical distribution of DTref_max per tile (approximation by a normal distribution), a mean value $(\mu)$ close to $\sim 4.5^{\circ} \mathrm{C}$ was observed with a standard deviation $(\sigma)$ $>1.5^{\circ} \mathrm{C}$ (Fig. 6). This result shows a global decrease of the average quality of Batch $\# 3$ after HHF test which can be attributed to several factors: decrease of CFC thermal conductivity due to degradation of the CFC matrix, corrosion of the inner channels, degradation of $\mathrm{CuCrZr}$ thermal conductivity, modification of the behaviour of the defect-free reference element. The analysis of the SATIR post-examination based on conventional statistical techniques used in quality control (i.e "Normality range with a level of confidence of $95 \%$ " corresponding to a range included within two reference standard deviation $\left(\sigma^{\text {ref }} \sim 1.5^{\circ} \mathrm{C}\right)$ away from the mean value of tested batch $\left(\mu \sim 4.5^{\circ} \mathrm{C}\right)$ ), meets an agreement close to $65 \%$ with the fatigue testing and confirms the presence of crack initiation and/or propagation during the fatigue tests. Visual inspections after HHF tests have also mostly correlated the hot spots observed under heat loads to initiation and/or propagation of cracks at the interface between CFC tiles and copper.

Recent development using a signal processing method [10], allowed to improve the defect detection capability of the SATIR test bed. This advanced method, based on a spatial image autocorrelation process SATIR thermo-signal by itself without using a defect-free element, helped the decision-making on tiles identified as questionable during SATIR inspection (Fig. 7, Cycle 100). The preliminary results show a better agreement with fatigue testing and underline the high likelihood of crack initiation during the high heat flux cycling.

\subsubsection{Discussion}

Considering the preliminary database from the GLADIS facility, the criteria of rejection associated with SATIR inspection before commissioning is reliable and validated (100\% of the rejected tiles after SATIR inspection were also detected as defective on GLADIS). However, the limited agreement observed between the pre-examination SATIR (or the screening GLADIS tests), and the HHF cycling seems to indicate the initiation/propagation of defects during the HHF test. This behaviour is clearly observed on several elements by the comparison of the SATIR inspection before (pre-examination) and after (post-examination) the HHF tests (Fig. 8). In addition, associated to SATIR inspection based on the so-called DTref criterion, a signal processing method without utilising a defect-free reference element, allowed to improve the defect detection of SATIR test bed.

However, some studies are still needed to better understand the detailed failure processes and to obtain information about defect topology. Furthermore, some improvements in the design may also enable to reduce the stress concentration at the interface between CFC tiles and heat sink and increase the safety margin.

\section{Conclusion}

Experience gained during manufacturing of Tore Supra actively cooled PFC led to the establishment of a qualification methodology for future PFC manufacture. It also provided a large experience of acceptance criteria using active infrared thermography (SATIR). Transient thermography offers the possibility of assessing the heat transfer capability of the whole component, thus being a fast and economical way to assess the acceptability of a component prior to its installation into a fusion machine.

The application of this methodology was used to define workable acceptance criteria for the CFC flat tile target elements of the W7-X divertor. 
Results obtained from qualification tests performed on mock-ups with artificial calibrated defects, demonstrated the capability of the active infrared thermography method to detect different types of defect in the size range 2-4 mm and enabled workable acceptance criteria to be proposed for the serial production. The application of this non-destructive acceptance test on pre-series elements, showed a good correlation with the initial screening at $6 \mathrm{MW} / \mathrm{m}^{2}$. However, some defects - not-detected as defective during NDE- appeared or propagated during cycling at nominal heat fluxes $\left(10 \mathrm{MW} / \mathrm{m}^{2}\right.$ in steady state). No tiles were detached during the thermal cycling tests. This behaviour led to an extension of the pre-series activities to improve the safety margin in the design and to achieve a successful series production of the W7-X divertor target elements. In addition, SATIR inspection before and after high heat flux tests in the ion beam GLADIS facility, enabled through an acceptance test based on a reference element to detect variability in terms of quality within the examined batch. This behaviour needs additional investigations such as metallographic study.

\section{References}

[1] J. Schlosser, A. Durocher, T. Huber, P. Garin, B. Schedler and G. Agarici, Experience feedback from high heat flux component manufacturing for Tore Supra, Fusion Engineering and Design vol. 56-57 (2001) pp. 309-313

[2] F. Escourbiac, A. Durocher, J. Schlosser, S. Fouquet, M. Missirlian and F. Cismondi, Actively cooled plasma facing components qualification and commissioning methodology, Proc. $4^{\text {th }}$ IAEA Technical Meeting, Gandhinagar (India), February (2005)

[3] A. Durocher, F. Escourbiac, A. Grosman, J. Boscary, M. Merola, F. Cismondi, X. Courtois, J.L. Farjon, M. Missirlian, J. Schlosser and R. Tivey, Advanced qualification methodology for Actively cooled high heat flux plasma facing components, Proc. $21^{\text {th }}$ IAEA conference, Chengdu (China), October 16-22 (2006)

[4] J. Boscary, H. Greuner, F.W. Hoffmann, B. Mendelevitch, K. Pfefferle and H. Renner, The development of divertor modules for W7-X, Physica Sripta T91, (2001), pp. 90-93

[5] J. Boscary, H. Greuner, B. Mendelevitch, B. Schedler, K. Scheiber, J. Schlosser and B. Streibl, Applied technologies and inspection for the W7-X pre-series target elements, Journal of Fusion Engineering and Design 75-79 (2005), pp. 451-455

[6] A. Durocher, R. Mitteau, V. Paulus-Martin and J. Schlosser, Interface quality control by infrared thermography measurement, Proc. $15^{\text {th }}$ World Conference on NDT, Roma, Italy, October 15-21 (2000)

[7] M. Missirlian, H. Traxler, J. Boscary, A. Durocher, F. Escourbiac, J. Schlosser, B. Schedler and P. Schuler, Infrared Thermography inspection methods applied to the target elements of W7-X, Proc. $24^{\text {th }}$ Symposium on Fusion Technology (SOFT), Warsaw (Poland), Sept. 10-15, 2006, to be published in Fusion Engineering and Design

[8] M. Rödig, I. Bobin-Vastra, S. Cox, F. Escourbiac, A. Gervash, A. Kapoustina, W. Kuehnlein, V. Kuznetsov, M. Merola, N. Nygren and D.L. Youchison, Testing of actively cooled mock-ups in several high heat flux facilities-An international round robin test, Journal of Fusion Engineering and Design 75-79 (2005), pp. 303-306

[9] H. Greuner, B. Boeswirth, J. Boscary, A. Plankensteiner and B. Schedler, High heat flux tests of the W7-X pre-series target element, Proc. $24^{\text {th }}$ Symposium on Fusion Technology (SOFT), Warsaw (Poland), Sept. 10-15, 2006, to be published in Fusion Engineering and Design

[10] F. Cismondi, B. Xerri, C. Jauffret, J. Schlosser, N. Vignal and A. Durocher, Analysis of SATIR test for qualification of high heat flux components: Defects detection and classification by signal-to-noise ratio maximisation, Presented to this conference 
"Standard \& Diagnostic " target elements
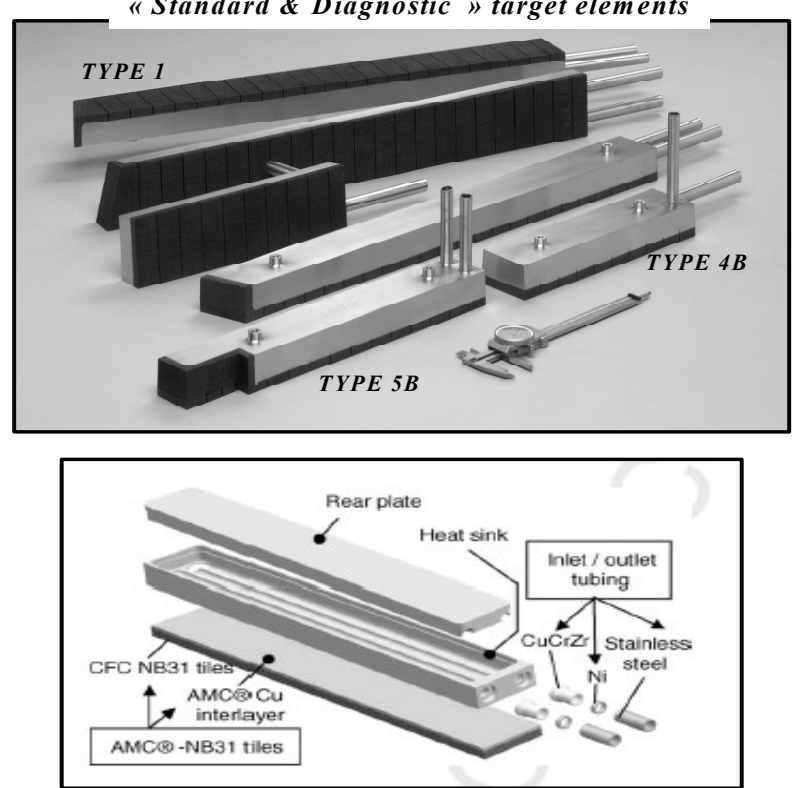

Fig 1. Target Elements of W7-X Divertor

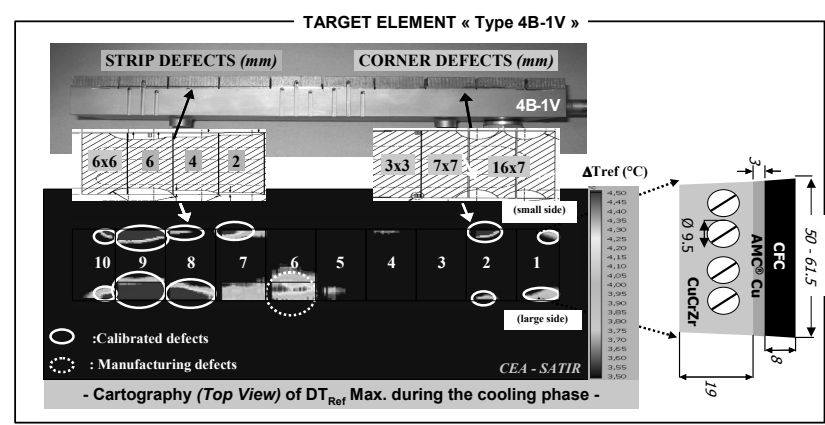

Fig 2. 4B-1V element (with artificial defects)

-IR cartography of DTref_max on SATIR- 


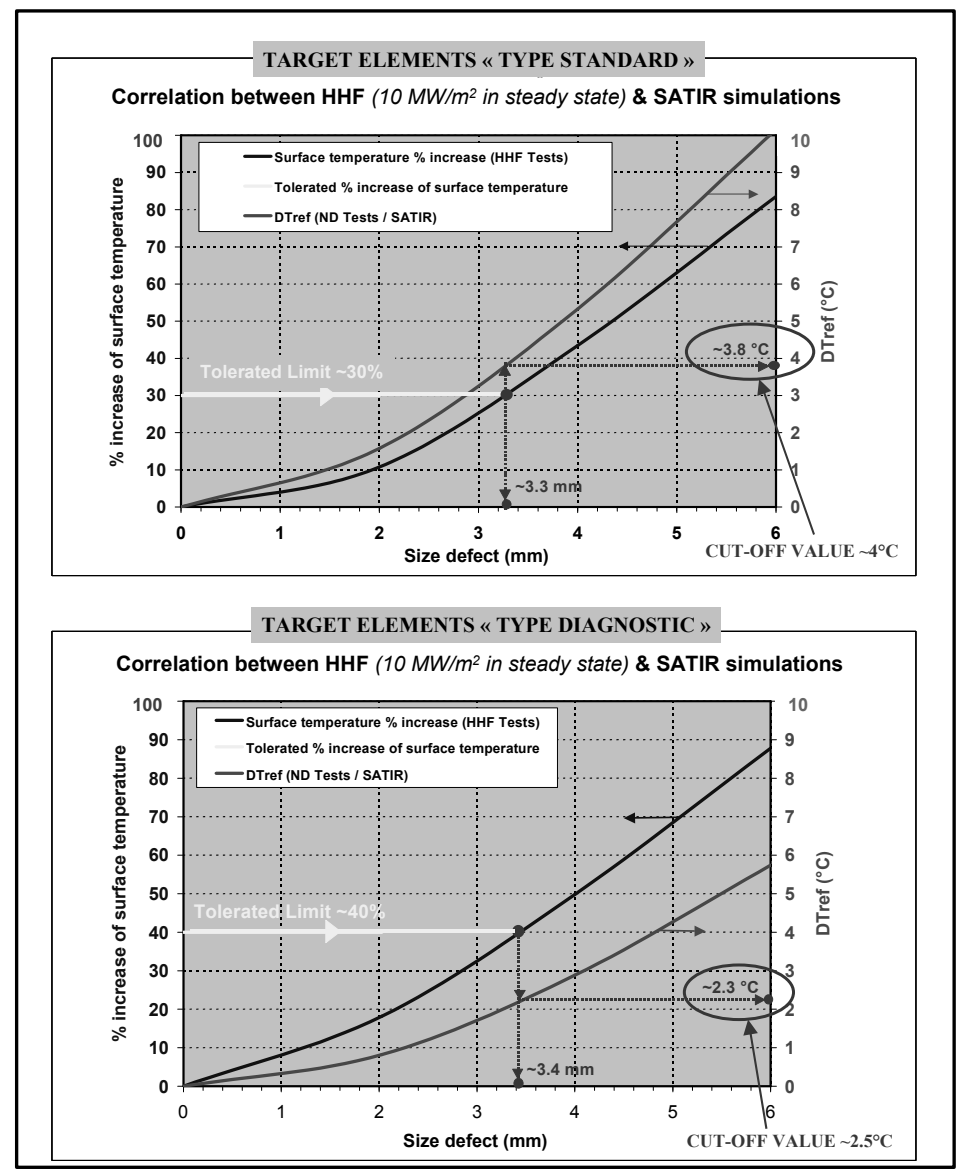

Fig. 3 Correlation between high heat flux testing and SATIR examinations

-"Cut-Off values" determination for the standard (i.e. small side) and diagnostic (i.e. large side) elements-

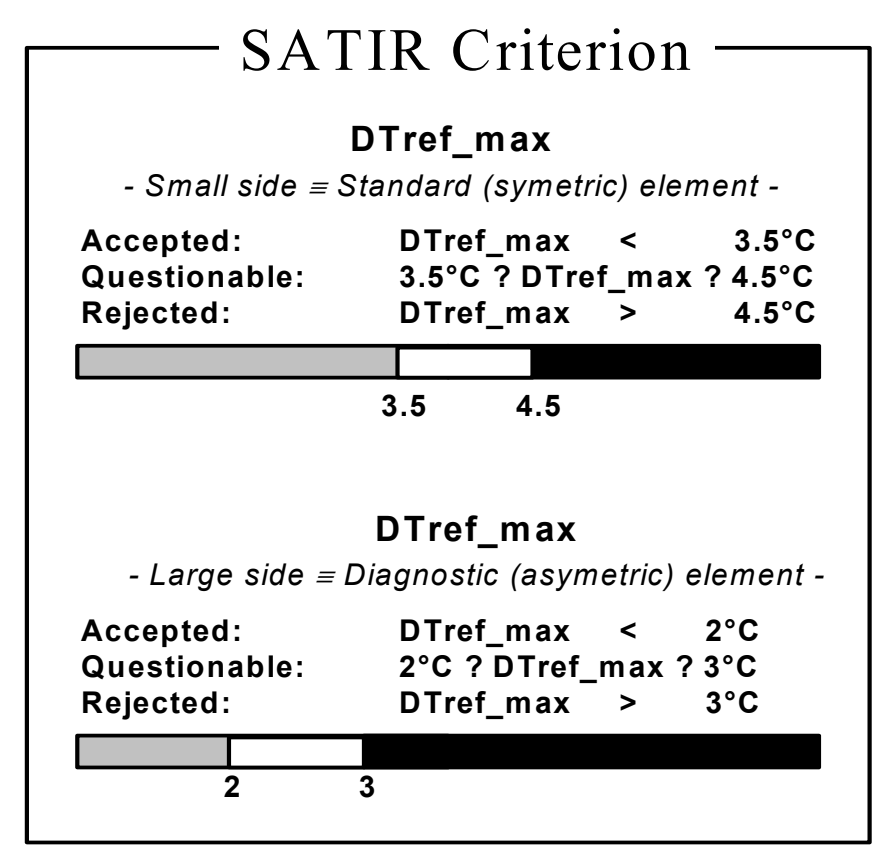

Fig. 4 SATIR Acceptance Criteria for W7-X target elements 


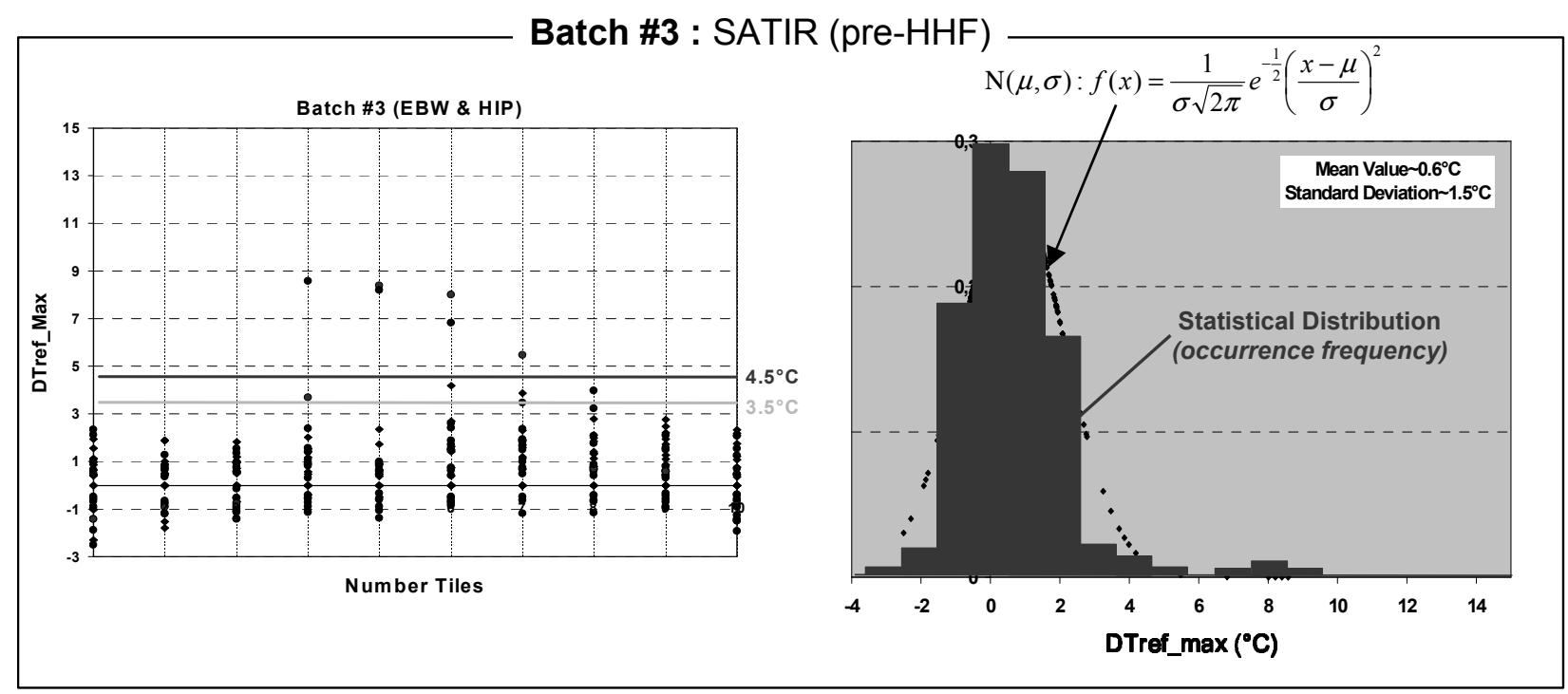

Fig. 5 Results of SATIR pre-examination on Batch \#3

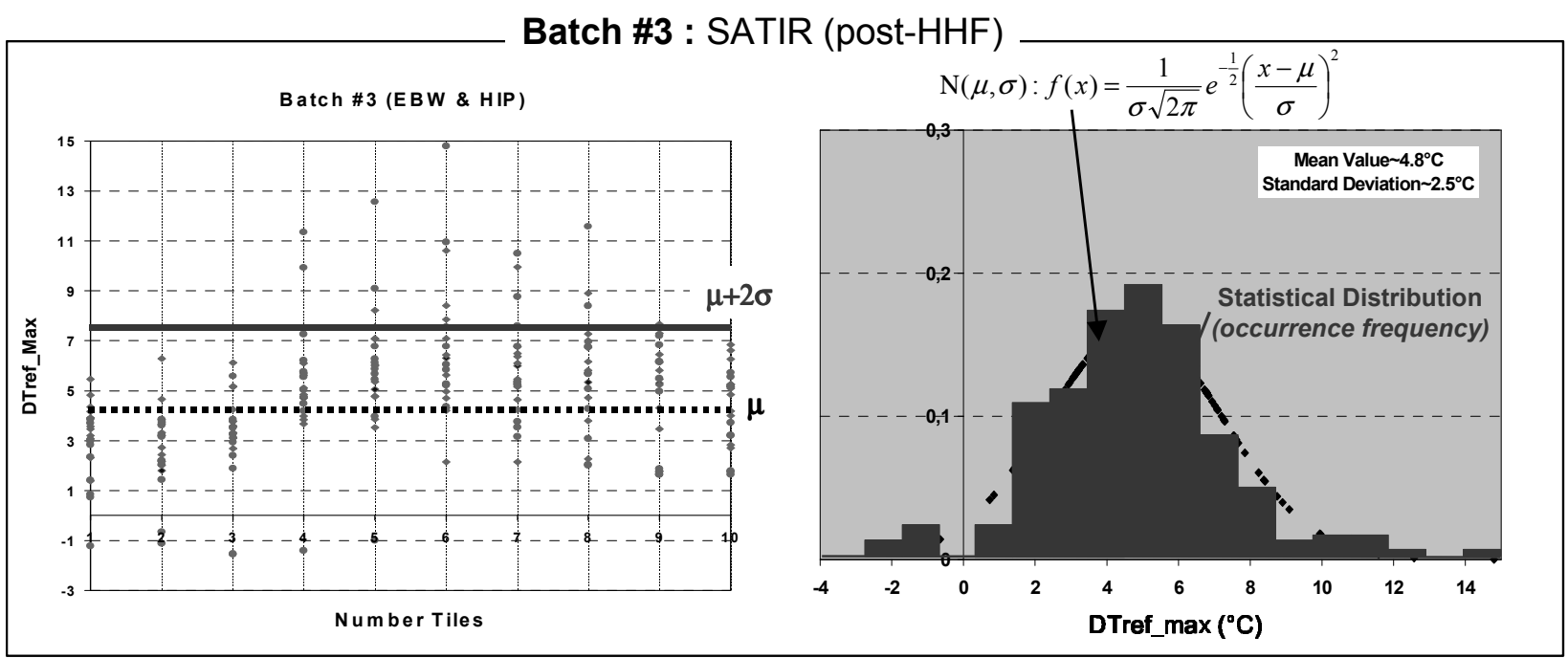

Fig. 6 Results of SATIR post-examination on Batch \#3

BATCH \#3: COMPARISON « SATIR NDE/GLADIS HHF TESTING »

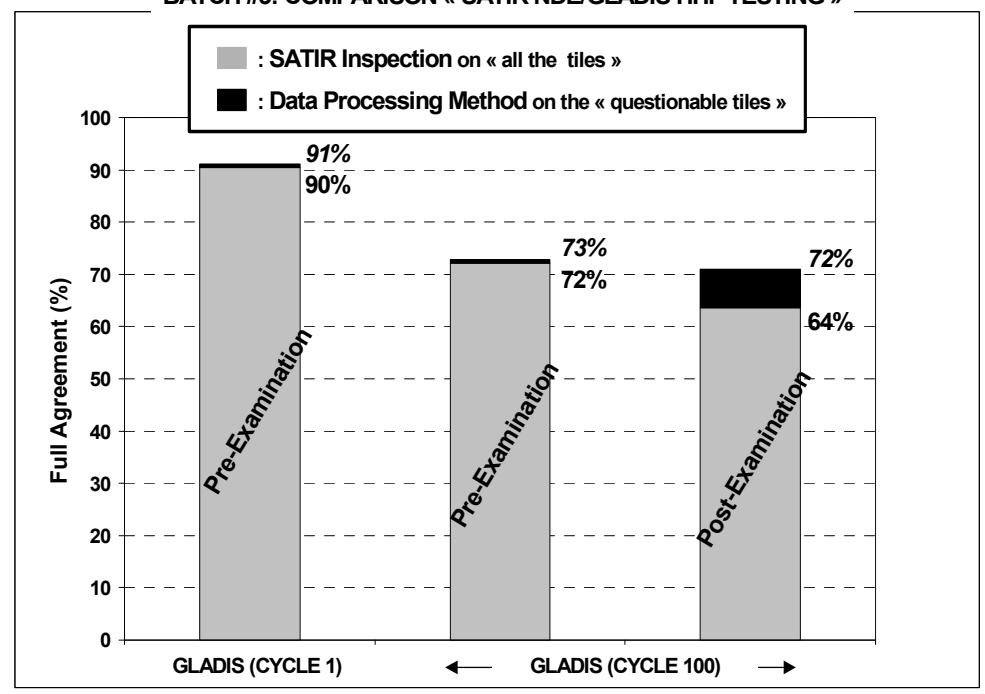

Fig. 7 Correlation between NDE (SATIR and Data Processing Method) and GLADIS HHF testing during the inspection of pre-series target elements (Batch \#3) 


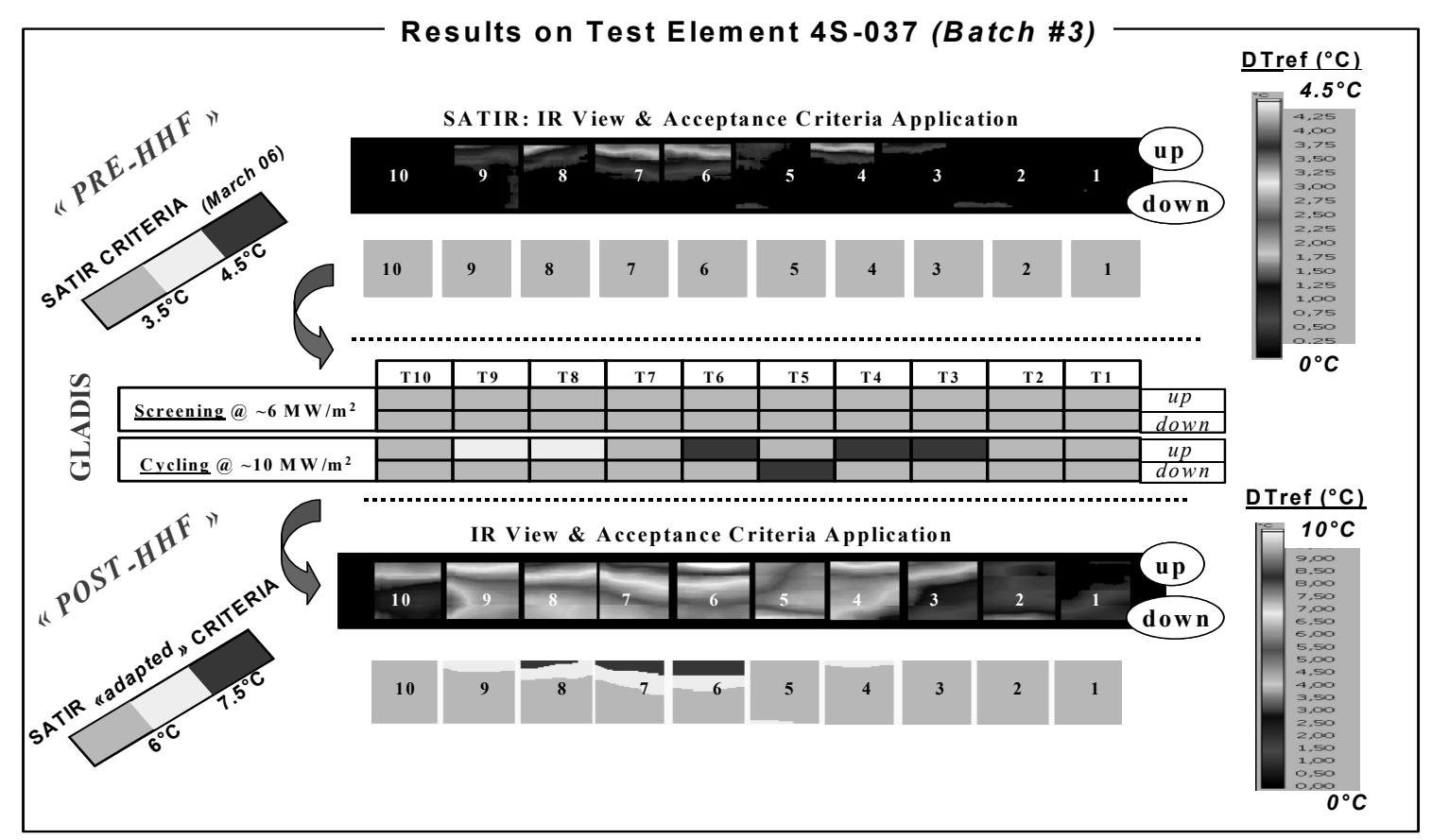

Fig 8. 4 S-037 element : SATIR and GLADIS results 